\title{
Design of Spectrally-Efficient Continuous Phase Modulations
}

\author{
Alan Barbieri, Dario Fertonani, and Giulio Colavolpe \\ Università di Parma, Dipartimento di Ingegneria dell'Informazione, Viale G. P. Usberti 181/A, Parma - ITALY \\ Email: giulio.colavolpe@unipr.it
}

\begin{abstract}
We investigate the spectral efficiency of continuous phase modulations (CPMs). To this end, we need an effective bandwidth definition for a CPM signal, whose power spectral density has in principle an infinite support. The definition we adopt is based on the spacing between adjacent carriers in a frequency division multiplexed CPM system. We consider the inter-channel interference, that depends on the channel spacing, and we evaluate the spectral efficiency achievable by a singleuser receiver in the considered multi-channel scenario. We then optimize the channel spacing with the aim of maximizing the spectral efficiency, showing that impressive improvements with respect to the spectral efficiency achieved without optimizing the channel spacing can be achieved.
\end{abstract}

\section{INTRODUCTION}

Continuous phase modulations (CPMs) form a class of constant envelope signaling, widely studied thanks to their appealing properties [1]. In particular, beside the robustness to non-linearities stemming from the constant envelope, CPMs exhibit an excellent power and spectral efficiency [1], [2]. To evaluate the spectral efficiency, which is an important quality figure for a modulation format, since it quantifies how many information bits per second can be loaded per unity of the available spectrum, a bandwidth definition is required. Nonetheless, although the power of a CPM signal is typically concentrated in a small portion of the spectrum [3], the power spectral density (PSD) has rigorously an infinite support, so that a suitable bandwidth definition must be assumed. The most commonly employed definition is that based on the power concentration, which defines the signal bandwidth as the bandwidth that contains a given fraction (being this fraction a parameter) of the overall power [4], [5]. A different definition is used in [6], which is based on the Carson's rule and exhibits a nice mathematical tractability. We also notice that some papers, as [7], investigated the information rate achievable by CPMs without accounting for the bandwidth and thus without giving any insight on the spectral efficiency. In this paper, we consider the spectral efficiency as the key quality factor.

A critical drawback of the above mentioned bandwidth definitions is that they are not prone to describe the impact of the power leakage outside the defined bandwidth in terms of interference caused to adjacent signals. As a matter of fact, from a communication system perspective, the final aim of any bandwidth definition is to evaluate the portion of the available spectrum taken up by a signal, so as to realize how far the adjacent channels should be placed from the signal under analysis when a frequency division multiplexed (FDM) system is considered. Based on this consideration, we consider a FDM-CPM transmission over a channel impaired by additive white Gaussian noise (AWGN), where all subchannels are equally spaced and adopt the same CPM format, so that the effective bandwidth of a CPM signal is implicitly defined as the separation between two adjacent channels. Then, we evaluate the ultimate information rate achievable when single-user detection is employed, that is, when each of the sub-channels is processed by a dedicated receiver which neglects the inter-channel interference (ICI)—we will use the terms "channels" and "users" interchangeably. For the considered scenario, which turns out to be an instance of mismatched decoding [8], the information rate can be evaluated by means of the simulation-based algorithm described in [9], as a function of the spacing between the channels (i.e., the effective bandwidth). Hence, a method for evaluating the ultimate achievable spectral efficiency results, which improves the existing methods since it accounts for the presence of ICI.

By means of the proposed framework, we show that, for any given CPM format, the achievable spectral efficiency is significantly larger than that resulting from the conventional approaches, in which a fixed, non optimized spacing is considered. In particular, it turns out convenient to consider frequency spacings between the channels much lower than those deriving from the above mentioned bandwidth definitions, which can provide a better tradeoff between degradation of the information rate due to the ICI, and usage of the available spectrum. Moreover, we show that the optimal spacing depends on the signal-to-noise ratio (SNR), that is like to say that the effective bandwidth of a CPM signal does depend on the SNR. The framework proposed in this paper can be used for selecting, based on information-theoretic arguments, a set of CPM formats suitable for a particular application, similarly to what has been done in [5] and [10] (see also [11]), where anyway the effect of ICI has been neglected-a comparison between such papers is fair since the same single-user receiver is considered.

The remainder of this paper is organized as follows. In Section II we give the system model, while in Section III we address the related ultimate performance limits. In Section IV we describe an algorithm for optimizing the achievable spectral efficiency, whose effectiveness is proved by the simulation results reported in Section V. Finally, Section VI gives some concluding remarks.

\section{SYSTEM MODEL}

The complex envelope of a CPM signal has the form [1]

$$
s(t ; \mathbf{x})=\sqrt{\frac{2 E_{S}}{T}} \exp \left\{j 2 \pi h \sum_{n} x_{n} g(t-n T)\right\}
$$


in which $E_{S}$ is the energy per symbol, $T$ is the symbol interval, $h=r / p$ is the modulation index ( $r$ and $p$ are relatively prime integers), the input symbols $\left\{x_{n}\right\}$, possibly correlated, take on values in the $M$-ary alphabet $\{ \pm 1, \pm 3, \ldots, \pm(M-1)\}$, and $\mathbf{x}=\left\{x_{n}\right\}$ denotes the input sequence. The function $g(t)$ is the phase-smoothing response and its derivative is the frequency pulse [1].

We consider a multi-channel FDM-CPM system, where all channels share the same CPM format and input alphabet. We assume that the input data streams are mutually independent, that all channels are received with the same power and are perfectly synchronized, i.e., no timing offsets are present between different channels (downlink assumption), and that the transmission channel only introduces AWGN, without any time or frequency selectivity. We remark that many of the above assumptions can be relaxed without affecting the main results of this paper. The overall complex baseband equivalent received signal yields

$$
y(t)=\sum_{k=-K}^{K} s\left(t ; \mathbf{x}^{(k)}\right) e^{j 2 \pi k F t}+w(t)
$$

where $F>0$ represents the spacing (in $\mathrm{Hz}$ ) between the carriers, $w(t)$ is a zero-mean circularly-symmetric white Gaussian process with PSD $2 N_{0}$, and $2 K+1$ sub-channels are present.

We consider single-user detection, that is, each of the subchannels is processed by a dedicated receiver which does not account for the ICI nor exchange any information with the receivers dedicated to the adjacent sub-channels. Without loss of generality, in the remaining of this paper we only consider single-user detection of the information stream $\mathbf{x}^{(0)}$. Let us rewrite (2) as

$$
y(t)=s\left(t ; \mathbf{x}^{(0)}\right)+w(t)+\sum_{k \neq 0} s\left(t ; \mathbf{x}^{(k)}\right) e^{j 2 \pi k F t}
$$

where the two different impairments experienced by the receiver, namely the background noise and the interference due to adjacent channels, are pointed out. Generally, it is assumed that the frequency spacing $F$ is large enough, such that the last term in (3) can be neglected in the bandwidth of interest. Here, we consider a more general approach, which consists of modelling the interference as a zero-mean circularlysymmetric white Gaussian process with PSD $2 N_{I}$, of course independent on the additive thermal noise-we point out that this approximation is exploited only by the receiver, while in the actual channel the interference is of course generated as in (3). Hence, the channel model assumed by the receiver is

$$
y(t)=s\left(t ; \mathbf{x}^{(0)}\right)+n(t)
$$

where $n(t)$ is a zero-mean circularly-symmetric white Gaussian process with PSD $2\left(N_{0}+N_{I}\right)$. As explained later, since no obvious way for choosing a value of $N_{I}$ from the system parameters exists, this choice should be based on computer simulations. We will show that, by means of a suitable tuning of the parameter $N_{I}$, the auxiliary channel (4) can describe with better and better approximation the real multiuser channel (3).

\section{Ultimate PeRformance Limits}

We are interested in evaluating the ultimate performance limits achievable by a single-user receiver designed for the auxiliary channel (4) when the actual channel is that in (3), in terms of information rate and spectral efficiency. This issue, which is an instance of mismatched decoding [8], cannot be addressed in closed form, but can be solved by exploiting the simulation-based method described in [9], which only requires the existence of an algorithm for exact maximum a posteriori (MAP) symbol detection over the auxiliary channel. In the considered case, the auxiliary channel (4) is an AWGN channel, for which algorithms for MAP symbol detection can be derived exactly either with a frontend based on the Rimoldi decomposition [2] and the BCJR algorithm [12], or by exploiting the Laurent decomposition [13] and a suitable forward-backward algorithm [14], [15]. We denote by $\mathbf{y}^{(0)}$ any sequence of samples that are sufficient statistics for the detection of $\mathbf{x}^{(0)}$, for example the samples at the output of the bank of filters of the Rimoldi-based frontend.

The simulation-based method described in [9] allows to evaluate the achievable information rate (AIR) for the mismatched receiver, i.e.,

$I\left(\mathbf{x}^{(0)} ; \mathbf{y}^{(0)}\right)=\lim _{N \rightarrow \infty} \frac{1}{N} E\left\{\log \frac{p\left(\mathbf{y}^{(0) N} \mid \mathbf{x}^{(0) N}\right)}{p\left(\mathbf{y}^{(0) N}\right)}\right\}\left[\frac{\text { bit }}{\text { ch. use }}\right]$

where we use the superscript $N$ to remark that a sequence is truncated to its first $N$ elements. In (5), $p\left(\mathbf{y}^{(0) N} \mid \mathbf{x}^{(0) N}\right)$ and $p\left(\mathbf{y}^{(0) N}\right)$ are probability density functions according to the auxiliary channel model (4), while the outer statistical average is with respect to the input and output sequences evaluated according to the actual channel model (3) [9]. Both $p\left(\mathbf{y}^{(0) N} \mid \mathbf{x}^{(0) N}\right)$ and $p\left(\mathbf{y}^{(0) N}\right)$ can be evaluated recursively through the forward recursion of the MAP detection algorithm matched to the auxiliary channel model [9]. Let us recall that the mismatched receiver can assure error-free transmissions when the provided information rate does not exceed $I\left(\mathbf{x}^{(0)} ; \mathbf{y}^{(0)}\right)$.

From a system viewpoint, the spectral efficiency, that is the amount of information transmitted per unity of time and per unity of bandwidth, is a more significant quality figure than the information rate. Hence, the derivation of the spectral efficiency for the considered system is given in the following. First, we notice that the overall information rate (in bits per channel use) achievable by the $2 K+1$ single-user receivers over the multi-user channel (2) is given by

$$
\sum_{k=-K}^{K} I\left(\mathbf{x}^{(k)} ; \mathbf{y}^{(k)}\right)
$$

where $\mathbf{y}^{(k)}$ is any sufficient statistic for the detection of $\mathbf{x}^{(k)}$, while the PSD of the multi-user signal is

$$
\sum_{k=-K}^{K} S_{X}(f-k F)
$$

$S_{X}(f)$ being the PSD of a single user. For increasingly large values of $K$ the boundary effects become negligible for both the overall information rate and the overall bandwidth, 


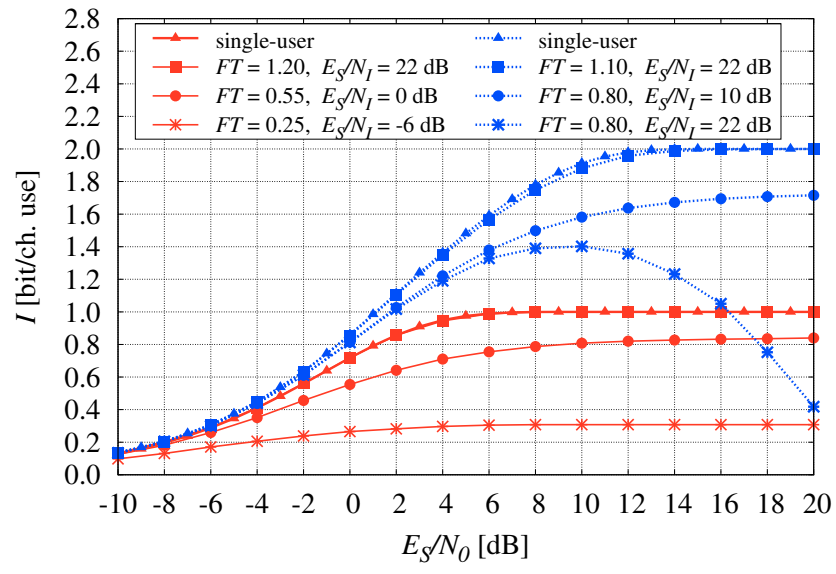

Fig. 1. Achievable information rate for an MSK modulation (red curves) and a quaternary 2-RC modulation with $h=1 / 5$ (blue curves).

hence, under the assumption of a large number of users, the achievable spectral efficiency (ASE) yields

$$
\eta=\frac{1}{F T} I\left(\mathbf{x}^{(0)} ; \mathbf{y}^{(0)}\right) \quad\left[\frac{\mathrm{bit}}{\mathrm{s} \cdot \mathrm{Hz}}\right]
$$

after simple manipulations. Hence, thanks to the assumption of a large number of users, we can keep focusing on the information stream $\mathbf{x}^{(0)}$ even for evaluating the ASE.

Some simulation results are reported in Fig. 1, which shows how the AIR varies with the value of $E_{S} / N_{0}$ when different values of the frequency spacing $F$ and the parameter $N_{I}$ are considered. As a benchmark, we added the corresponding single-user curves (equivalent to the mismatched case with infinite $F$ ). These results refer to a binary 1-REC modulation with $h=1 / 2$, often referred to as minimum-shift keying (MSK) modulation, and a quaternary 2-RC modulation with $h=1 / 5-L$-REC and $L$-RC denote respectively rectangular and raised cosine frequency pulses of length $L T$ [1]. Fig. 1 gives a quantitative evidence of the fact that the lower the value of $F$, the larger the interference due to the adjacent channels, and therefore the smaller the resulting AIR. In particular, when the value of $F$ is such that about the $99 \%$ of the signal power is concentrated in the bandwidth $[-F / 2, F / 2]$ [5], [16], namely when $F T=1.2$ for the MSK modulation and $F T=1.1$ for the quaternary 2-RC modulation with $h=1 / 5$, the impact of the interference is nearly null. Also, Fig. 1 clarifies the impact of the parameter $N_{I}$, showing that, when it is significantly underestimated, the mismatch between the auxiliary channel and the actual one is such large that the AIR can decrease as the SNR increases. We remark again that the values of information rate shown in Fig. 1 are achievable by a MAP single-user receiver designed for the channel model (4).

On the other hand, very interesting insights are given by the results reported in Fig. 2, which shows the ASE corresponding to the same multi-user systems as in Fig. 1. These results clarify that the values of $F$ providing the best AIR are not those providing the best ASE, and thus that a careful design strategy, when the spectral efficiency is the key quality figure, should trade an intentional degradation

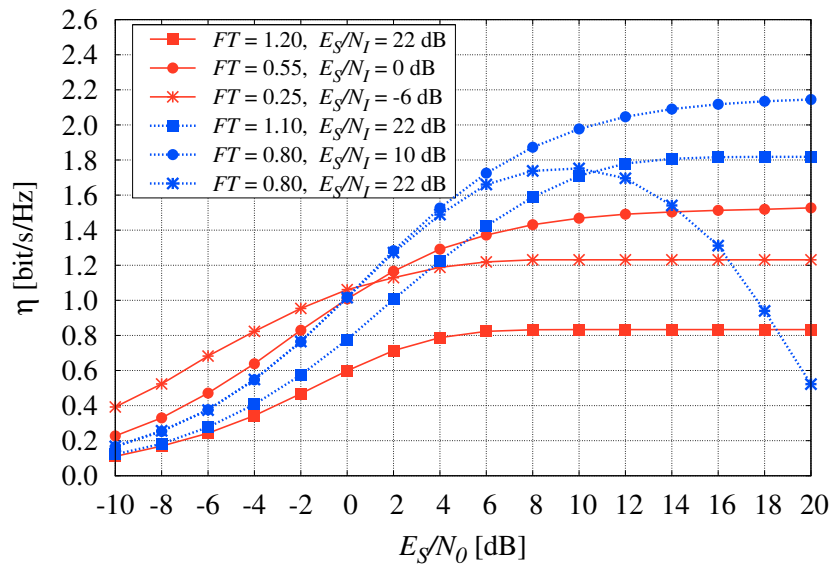

Fig. 2. Achievable spectral efficiency for an MSK modulation (red curves) and a quaternary 2-RC modulation with $h=1 / 5$ (blue curves).

in AIR for a larger ASE. For instance, in the case of the MSK modulation, the choice $F=1.2$, based on the $99 \%$ of the signal power, provides a spectral efficiency dramatically lower than the choice $F=0.55$. This fact confutes various previous works as [5], [6], [16], where the search for spectrally efficient schemes was carried out without exploiting the above mentioned tradeoff between AIR and ASE. An optimization algorithm able to exploit it is presented in the next section.

\section{Optimization OF THE SPECTRAL EFFICIENCY}

Our aim is to find, for a given CPM format, the frequency spacing $F$ that provides the largest ASE. As clear from the results reported in Fig. 2, we should expect that the optimal spacing does depend on the SNR. Fig. 3, which refers to the same quaternary modulation as in Fig. 1 and Fig. 2, gives more insights on this fact, suggesting that, as the signal-tonoise ratio increases, not only the ASE increases, but also the optimal spacing increases as well. The fact that at low values of the SNR the spacing can be "safely" reduced can be explained by realizing that an increase of the interferers power does not significantly affect the AIR as long as it is lower than the background noise power. The results reported in Fig. 3 were obtained by setting $N_{I}=0$, but a different choice would not affect the qualitative behavior of the system.

The properties of the function $\eta(F)$ cannot be easily studied in closed form, ${ }^{1}$ but it reads clear, by physical arguments, that it is bounded, continuous in $F$, and tends to zero when $F \rightarrow 0$ or $F \rightarrow \infty$. Hence, the function $\eta(F)$ has a maximum valueaccording to Fig. 3, we could also conjecture that there are no local maxima other than the global maximum. Formally, for a given CPM format and a given value of $E_{S} / N_{0}$, the optimization problem consists of finding the maximal ASE

$$
\begin{aligned}
\eta_{\mathrm{M}}\left(E_{S} / N_{0}\right) & =\max _{F>0, N_{I} \geq 0} \eta\left(F, N_{I}, E_{S} / N_{0}\right) \\
& =\max _{F>0, N_{I} \geq 0} \frac{I\left(F, N_{I}, E_{S} / N_{0}\right)}{F T}
\end{aligned}
$$

\footnotetext{
${ }^{1}$ Although the functions AIR $I(\cdot)$ and ASE $\eta(\cdot)$ depend on various system parameters, in the following we will only explicitly indicate the parameters of interest for the relevant discussion.
} 


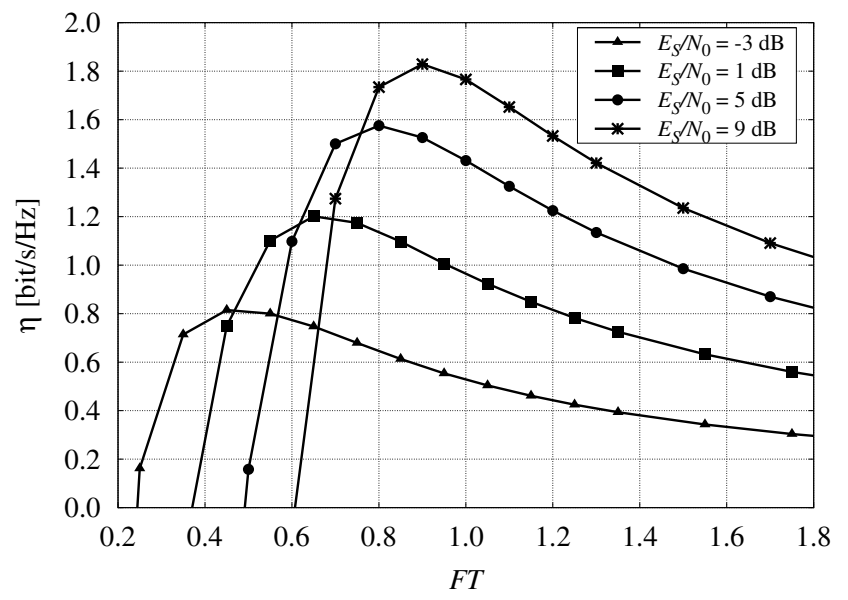

Fig. 3. Achievable spectral efficiency as a function of the spacing $F$, for a quaternary 2-RC modulation with $h=1 / 5$, when $N_{I}=0$.

which can be solved by evaluating $\eta\left(F, N_{I}, E_{S} / N_{0}\right)$ on a grid of values of $F$ and $N_{I}$ (coarse search), followed by an interpolation of the obtained values (fine search). However, since we found that the dependence of the ASE on the value of $N_{I}$ is rather coarse, and in order to speed up the simulation process, we decided to choose a single value $\hat{N}_{I}$ of $N_{I}$ for each CPM format, by means of the asymptotic (in the signalto-noise ratio) joint optimization

$$
\hat{N}_{I}=\underset{N_{I}}{\operatorname{argmax}} \max _{F>0} \lim _{E_{S} / N_{0} \rightarrow \infty} \eta\left(F, N_{I}, E_{S} / N_{0}\right) .
$$

Hence, we remark that the optimized spectral efficiencies shown in the next section can be (slightly) improved by jointly optimizing $F$ and $N_{I}$ for each value of $E_{S} / N_{0}$, as in (7).

So far, we addressed the optimization problem for a given value of $E_{S} / N_{0}$. On the other hand, a more significant measure of the SNR than $E_{S} / N_{0}$ is given by $E_{b} / N_{0}$, being $E_{b}$ the energy per information bit, for which the following equation holds

$$
E_{S}=I\left(E_{S}\right) E_{b} .
$$

In order to solve the optimization problem for a given value of $E_{b} / N_{0}$, we employed the following algorithm. First, a set of values of the spacing $F_{1}<F_{2}<\ldots<F_{N}$ are chosen, depending on the CPM format, and, for each $n=1,2, \ldots, N$, the AIR is evaluated for a set of suitable values of $E_{S} / N_{0}$. Once evaluated these values, they are interpolated by means of a cubic spline to find a regular expression of $I\left(F_{n}, E_{S} / N_{0}\right)$. Then, given a value of $E_{b} / N_{0}$, the following fixed point problem is solved in $E_{S} / N_{0}$

$$
\frac{E_{S}}{N_{0}}=I\left(F_{n}, \frac{E_{S}}{N_{0}}\right) \frac{E_{b}}{N_{0}} .
$$

and the AIR corresponding to the solution is denoted by $I\left(F_{n}, E_{b} / N_{0}\right)$. (Clearly, since there exists a lower bound on the achievable value for $E_{b} / N_{0}$, the above problem has no solution when unachievable values are considered.) Finally, a regular function $I\left(F, E_{b} / N_{0}\right)$, with $F \in\left[F_{1} ; F_{N}\right]$, is obtained by interpolating the samples $I\left(F_{n}, E_{b} / N_{0}\right)$, and this regular function allows to easily solve the maximization problem.

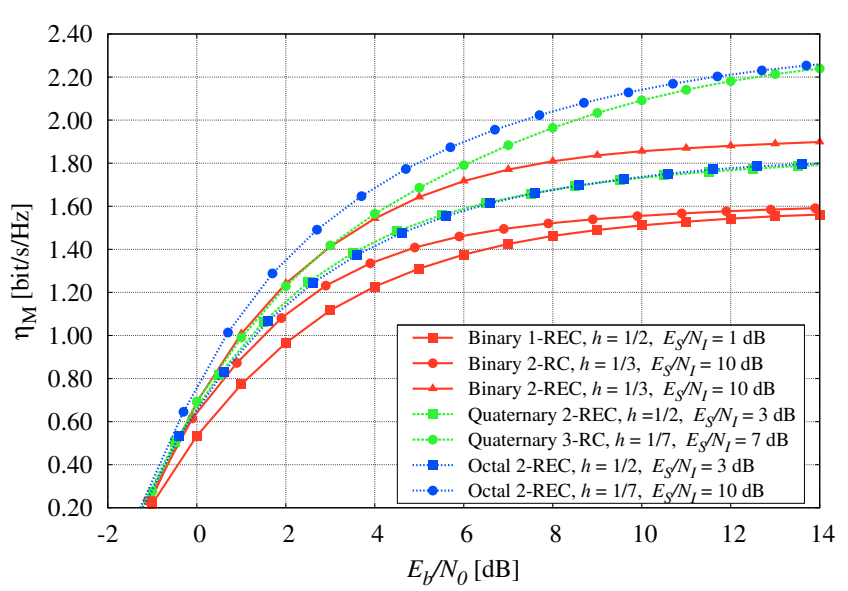

Fig. 4. Maximal achievable spectral efficiency as a function of $E_{b} / N_{0}$, for a set of binary, quaternary, and octal CPM schemes.

\section{NumericAl Results}

Some outcomes of computer simulations implementing the proposed optimization algorithm are reported and discussed in the following. In all of them, the optimization is carried out with respect to $E_{b} / N_{0}$.

Fig. 4 shows the maximal ASE $\eta_{M}$ as a function of $E_{b} / N_{0}$, for a set of CPM formats whose details are reported in the key. Similar comparisons can be used to select one or more CPM formats out of a given set, according to an information theoretic performance metric (namely, the maximal spectral efficiency achievable by single-user detection). An interesting conclusion, confirmed by other simulation results not reported here, is that, once all the other parameters are set, modulations based on a REC frequency pulse always outperform the corresponding modulations based on a RC frequency pulse. This fact was unexpected, since it is widely believed that the RC frequency pulses provide better spectral properties [1], [5], [6], [10], [11], and is discussed later. In Fig. 5 the optimized normalized spacing $F T$ is shown for a subset of the CPM formats investigated in Fig. 4. This figure confirms a behavior conjectured in Section IV, namely the increase of the optimized spacing with the signal-to-noise ratio. On the other hand, although increasing with the SNR, the optimal spacings are much lower than the values related to the $99 \%$ of the signal power, again proving that a tradeoff between AIR and ASE is required. Moreover, we notice that the optimal spacing of the REC-based modulation is much smaller than that of the RC-based one. This explains why REC-based formats achieve higher spectral efficiencies than their RCbased counterpart. This behavior is probably due to a larger robustness to ICI of the REC-based schemes with respect to those based on RC-based pulses, and is under further investigation - the robustness to intentional ICI was indeed not accounted for in the classical literature [1] nor in recent works as [5], [6], [10], [11], [16].

In [17] it was shown that the asymptotic (i.e., for vanishing small noise power) spectral efficiency of a given CPM format can be considerably improved with a suitable shaping of the input sequence. Thanks to its mathematical tractability, the 


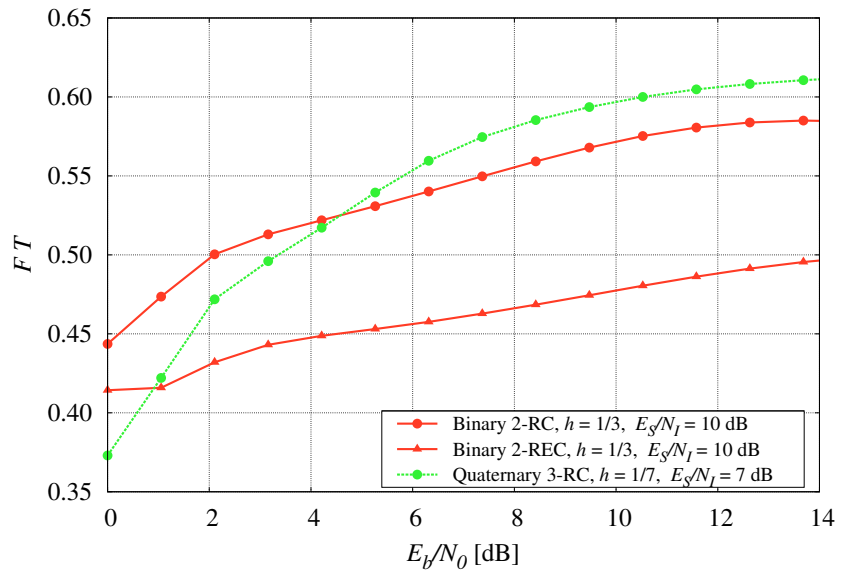

Fig. 5. Optimized normalized spacing as a function of $E_{b} / N_{0}$, for a selected set of CPM formats.

Carson's bandwidth was used to define the spectral efficiency. We considered one of the CPM formats studied in [17], namely an octal 2-REC with $h=1 / 2$, and we used the proposed method to evaluate the maximal ASE (through optimization of the spacing) when the optimized input distribution, proposed in [17], is used. For the above mentioned CPM format, in Fig. 6 we compare the maximal ASE for a quaternary and octal independent and uniformly distributed (i.u.d.) input, as well as for octal input optimized according to [17]. >From the figure, it is clear that input optimization does not lead to any improvement in terms of maximal spectral efficiency, with respect to i.u.d. octal input. Even an i.u.d. quaternary input achieves the same performance. We notice that the asymptotic value of about $1.8 \mathrm{bit} / \mathrm{s} / \mathrm{Hz}$ obtained with the three different input formats, is obtained with completely different values of the spacing - the relevant figure is not reported here due to the lack of space. In conclusion, when a suitable tradeoff between AIR and ASE is exploited, the input optimization proposed in [17] does not lead to any performance improvement.

\section{CONCLUSIONS}

We have considered a FDM-CPM multi-user system and we have evaluated the information rate achievable by a mismatched receiver, namely a single-user MAP detector employed in the considered multi-user scenario. We have proposed an algorithm for selecting the system parameters providing the best spectral efficiency. Several insights have been given which confute well established beliefs found in the literature. In particular, we have shown that a suitable tradeoff between AIR and ASE is required, that the REC-based modulations outperform the RC-based ones, and that the input optimization proposed in [17] is not effective in the considered multi-user scenario. As a future work, we will investigate if the use of the Markovian sources proposed in [16] can further improve the achievable spectral efficiency.

\section{ACKNOWLEDGMENT}

This work is funded by the European Space Agency, ESA-ESTEC, Noordwijk, The Netherlands, under contract no. 19370/05/NL/JD.

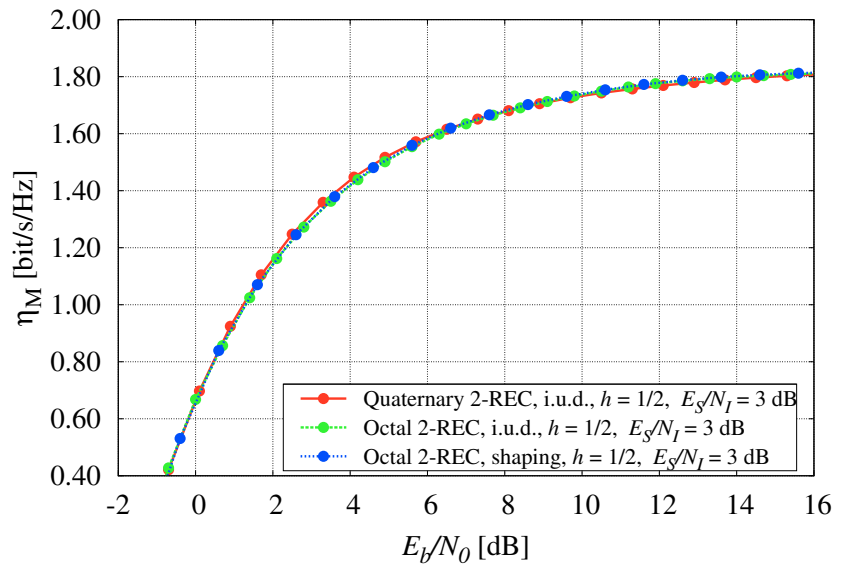

Fig. 6. Maximal achievable spectral efficiency as a function of $E_{b} / N_{0}$, for quaternary and octal 2-REC modulations with $h=1 / 2$.

\section{REFERENCES}

[1] J. Anderson, T. Aulin, and C.-E. Sundberg, Digital Phase Modulation. New York: Plenum Press, 1986.

[2] B. E. Rimoldi, "A decomposition approach to CPM," IEEE Trans. Inform. Theory, vol. 34, pp. 260-270, Mar. 1988.

[3] T. Aulin, N. Rydbeck, and C.-E. W. Sundberg, "Continuous phase modulation-Part II: partial response signaling," IEEE Trans. Commun., vol. 29, pp. 210-225, Mar. 1981.

[4] K. Padmanabham, S. Ranganathan, S. P. Sundaravaradhan, and O. M. Collins, "General CPM and its capacity," in Proc. IEEE International Symposium on Information Theory, pp. 750-754, Sept. 2005.

[5] S. Benedetto, G. Montorsi, A. Perotti, and A. Tarable, "Optimization of CPM pragmatic capacity," in Proc. IEEE Global Telecommun. Conf., Nov. 2007.

[6] C. H. Kuo and K. M. Chugg, "On the bandwidth efficiency of CPM signals," in Proc. IEEE Military Comm. Conf. (MILCOM), pp. 218-224, Oct.-Nov. 2004.

[7] S. Benedetto, G. Montorsi, A. Perotti, and A. Tarable, "A pragmatic approach to coded continuous-phase modulation," in Proc. Inform. Theory and Appl. Workshop, pp. 36-40, Jan.-Feb. 2007.

[8] N. Merhav, G. Kaplan, A. Lapidoth, and S. S. Shitz, "On information rates for mismatched decoders," IEEE Trans. Inform. Theory, vol. 40, pp. 1953-1967, Nov. 1994

[9] D. M. Arnold, H.-A. Loeliger, P. O. Vontobel, A. Kavčic̀, and W. Zeng, "Simulation-based computation of information rates for channels with memory," IEEE Trans. Inform. Theory, vol. 52, pp. 3498-3508, Aug. 2006.

[10] A. Graell i Amat, C. A. Nour, and C. Douillard, "Serially concatenated continuous phase modulation for satellite communications," submitted to IEEE Trans. Commun., July 2007.

[11] A. Graell i Amat, C. A. Nour, and C. Douillard, "Serially concatenated continuous phase modulation with extended BCH codes," in Proc. IEEE Inf. Th. Work. on Inf. Th. for Wir. Net., pp. 1-5, July 2007.

[12] L. R. Bahl, J. Cocke, F. Jelinek, and J. Raviv, "Optimal decoding of linear codes for minimizing symbol error rate," IEEE Trans. Inform. Theory, vol. 20, pp. 284-287, Mar. 1974.

[13] P. A. Laurent, "Exact and approximate construction of digital phase modulations by superposition of amplitude modulated pulses (AMP)," IEEE Trans. Commun., vol. 34, pp. 150-160, Feb. 1986.

[14] G. Colavolpe and A. Barbieri, "On MAP symbol detection for ISI channels using the Ungerboeck observation model," IEEE Commun. Letters, vol. 9, pp. 720-722, Aug. 2005.

[15] A. Barbieri and G. Colavolpe, "Simplified soft-output detection of CPM signals over coherent and phase noise channels," IEEE Trans. Wireless Commun., vol. 6, pp. 2486-2496, July 2007.

[16] A. Barbieri, A. Cero, A. Piemontese, and G. Colavolpe, "Markov capacity of continuous phase modulations," in Proc. IEEE International Symposium on Information Theory, June 2007.

[17] C. H. Kuo and K. M. Chugg, "Improving the bandwidth efficiency and of CPM signals via shaping and iterative detection," in Proc. IEEE Military Comm. Conf. (MILCOM), pp. 596-602, Oct. 2005. 